\title{
Fractal Dimension based SAR Image Sparse Degrees Estimation
}

\author{
Hua Bo ${ }^{a}$, Haiyun $\mathrm{Gu}^{\text {b}}$, Lei Ren, Hong Xie \\ Department of Electrical Engineering, Shanghai Maritime University, Shanghai, 210306, China \\ aemail: huabo@shmtu.edu.cn, bemail: hygu@shmtu.edu.cn
}

Keywords: Compressive sensing, Measurement matrix, Sparsity, Fractal dimension, SAR image

\begin{abstract}
Compressed sensing is surprisingly predicts that images, which allow a sparse representation by a suitable basis or a frame, can be recovered from what was previously considered highly incomplete linear measurements by using efficient algorithms. It is a novel research area of SAR images processing. As a result of the design of the observation matrix estimate depends on the image sparse degree, therefore, the estimation precision of the image sparse degree is an important factor to whether can accurate reconstruction images. In this paper, the relationship between the degrees of SAR image sparse and fractal dimension is researched. The experimental results show that the SAR image fractal dimension is proportional to the sizes of the observation matrix, which can be used to set the parameters of the observation matrix.
\end{abstract}

\section{Introduction}

SAR image processing is a novel research area due to the nature of their day and all-weather. Compressed sensing theory used to study the SAR image data acquisition and compression, have very important significance. Compressed sensing theory is proposed based on image sparse, under the condition of sparse image satisfaction, and can be far less than the Nyquist frequency of image sampling, can recover higher accuracy of reconstructed data with appropriate reconstruction algorithms.

If an image $\mathrm{f}$ with a length $\mathrm{N}$ is sparse under the orthogonal basis, it can be expressed in the formula $\mathrm{f}=\psi$ a, with a sparse matrix a. Observation image y is obtained by observation matrix , and $\mathrm{y}=\phi \psi \mathrm{a}$, $\mathrm{y}$ have the length $\mathrm{M}$ and $\mathrm{M}<<\mathrm{N}$.

$\mathrm{Y}$ is used to recover the image $\mathrm{f}$ by using the compressed sensing theory, its essence is to solve an abnormal condition equation, which equation size $\mathrm{M}$ is less than variable size $\mathrm{N}$. This equation is supposed to have infinite solutions but it just have a one solution under the condition named limited isometric conditions [1] [2], In this case, the sparse degree $\mathrm{k}$ meet the formula (1).

$\mathrm{M} \approx \mu^{2} \cdot \mathrm{K} \cdot \log \mathrm{N}$

Usually, for $M=4 \mathrm{k}$. For the accuracy of image reconstruction is depends on the sparse degree $\mathrm{k}$, its estimation has important significance.

At present, the image sparse research mainly in the image form and image model two aspects, the sparse model including Markov models, frequency models and dictionary models and so on. The analysis of image form was including the following works: literature [3] estimated the $\mathrm{k}$ by using the image complexity and edge characters; literature [2] used wavelet to express the $\mathrm{k}$, literature [5] proposed a PCA based estimate method and etc. Meanwhile, the analysis of image model research works was focus on establish the image model of sparse express, the model parameters show the sparsity of images. Images were mapping to a new coordinate system, where they have $\mathrm{k}$ sparse parameters. These methods are realizable. For example, an image having time-varying information just has a few frequency components. This fact is this image is sparse in frequency domain. Wavelet is also an effective method to reduce the image components. All these methods are depends on the mapping space, and almost these methods have more complexity. This paper proposed a novel method to estimate the $\mathrm{k}$ by using computing the image fractal dimension. This paper reveals the relationship between fractal and sparse degree $\mathrm{k}$, and obtained the mathematics description. This method is independent on the function basis and has a low complexity. 


\section{Fractal Dimension}

Fractal has not a clear definition until now. Mandelbrot given a description about fractal: the similar of part and overall. Except this rule-fractal, anomaly fractal means the statistical significance similarity. In mathematics fractal's research on the modal similarity, but in practice it is expanded to results, information, function, time and energy and etc.

Dimension is an important character to describe the complexity or convolution-degree of object of research. Hausdoff and Besicovitch expended the integer dimension to decimals and firstly presented the continuous space concept in 1919. Meanwhile, fractal dimension has many definitions and computing methods, for example, Similarity Dimension, Compass Dimension, Box-counting Dimension and Hausdoff Dimension etc. All these methods compute the rate of part and entire for finding the similarity.

Differential Box Counting is major used at present. Its process is as follows: at first segment the image of $\mathrm{M} \times \mathrm{M}$ to blocks of $\mathrm{L} \times \mathrm{L}, \mathrm{r}=\mathrm{L} / \mathrm{M}$; in the block of $(i, j)$ th the minimum gray value and the maximum gray value fall in the kth and lth box, definite $n_{r}(i, j)=l-k+1$ is the number of block (i,j)th; then compute all the box's numbers: $N_{r}=\sum_{i_{i}, j} n_{r}(i, j)$. In the end, definite the fractal dimension of this image:

$$
\mathrm{D}=\lim \frac{\log \left(\mathbb{N}_{r}\right)}{\log \left(\frac{1}{7}\right)}
$$

Choose a group L to compute and obtain the D by using linear fitting. As the process shows that the fractal dimension expresses the image pattern complexity and the gray value change rule but the image similarity.

Entropy is used to express the image gray change rule and has the meaning of chaos degree. Thus, fractal dimension and entropy are equivalent concept in statistical property. An experiment is designed to verify above-mentioned assumption. 88 SAR images were computed their fractal dimension and entropy, the result as shown in the Figure 1.

Figure 1 (a) shows the original result, (b) shows the result after change the entropy scale for showing the consistent variation trend more clearly. Scale doesn't impact the conclusion. Entropy and fractal dimension all can be used to compute the image complexity, but fractal dimension computing has more robust result and more quickly computing speed. In this paper fractal dimension is used to estimate the image sparseness.
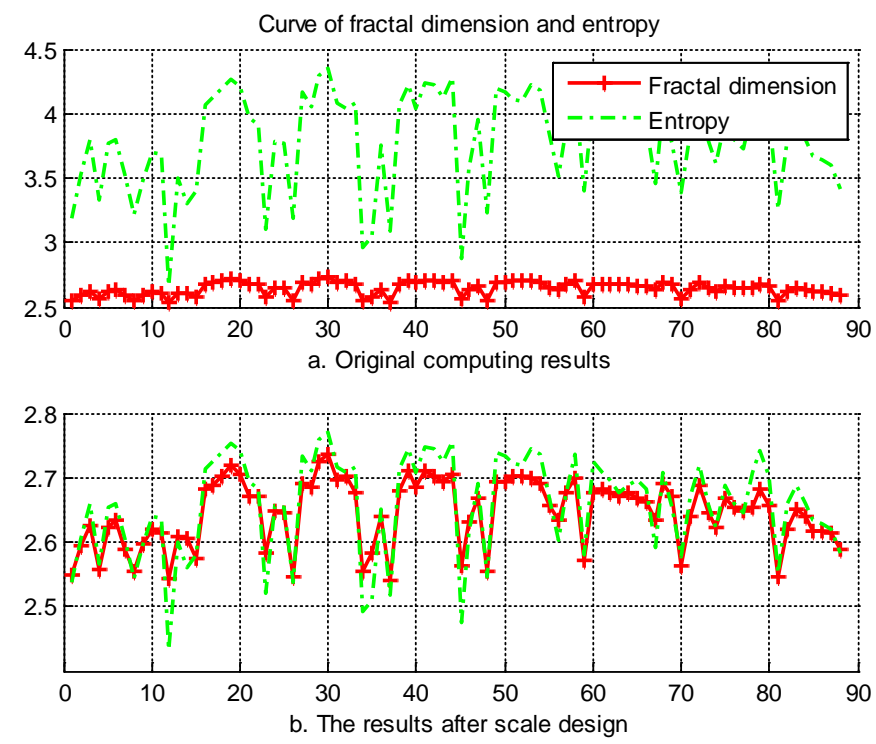

Fig.1. SAR Image’s Fractal dimension and Entropy

\section{Relationship between reconstruction precision and fractal dimension}

Compute the correlation curve of fractal dimension and reconstruction precision to research the relationship of fractal dimension and image sparse. Reconstruct some images with different sparse 
degree to obtain some relationship curves of sparsity and signal-noise rate of images. Given same signal-noise rate, the sparsity of images can be computed.

If the product $\phi \psi_{\text {of }}$ observation matrix and orthogonal basis satisfies limited equidistance condition, reconstruction image's signal-noise rate has a direct proportion to the observation matrix length $M$, this result as show as figure 2 . Thus if the reconstruction precision was confirmed, the observation matrix length $M$ was confirmed too. This method has great significance for SAR images processing because the mass data.

This paper selected 88 images to research. Their fractal dimensions were computed and signal-noise rate under different observation matrix length M. Six curves were shown in Figure 2.

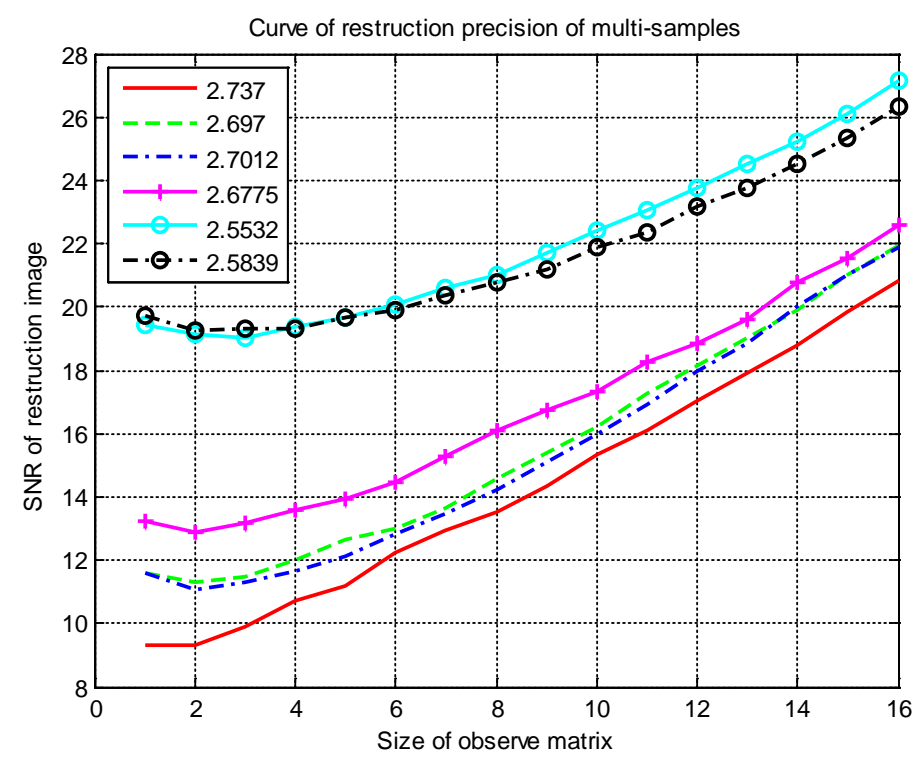

Fig.2. The multi-sample SNR curve of different observation matrix size

As the Figure 2 shown, the images having different sparsity degree need relevantobservation matrix length $\mathrm{M}$ to obtain same SNR. The fractal dimension values have same rule with sparsity degree. This experiment verifies the hypothesis above.

\section{Fractal dimension based SAR image sparsity estimation algorithm}

Fractal dimension calculation is simpler and contains understandable physical significance, comparing to current estimation algorithm of sparsity of images.Fractal dimension represents the distribution of patterns of reconstructed images. In this paper, Differential Box Counting algorithm is used to calculate fractal dimensions, number of patterns contained in the images and the complexity of distribution of patterns.

The method of estimation of sparsity:

1. Choose samples which have size 256 and then calculate the fractal dimensions of chosen images.

2. Reconstruct the samples based on different assumptions on sparsity. When $\mathrm{K}$ reaches maximum 64, the size of the measurement matrix is the same as original images which is the maximum value of reconstruction.

3. Reconstruct the samples which are compressed based on different sparsity, by using OMP reconstruction algorithm. At the same time, calculate the error during construction and the signal-noise ratios of reconstructed images.

4. Plot the relation curves between signal-noise ratio and sparsity $\mathrm{K}$ and then find the sparsity $\mathrm{K}$ corresponding to signal-noise ratio 20db.(decimal places are allowed for the simulation accuracy later in the paper)

5. Repeat step 1-4 on all 88 samples and collect data. Plot a line chart based on these data.(Figure 3(a))

6. Simulate a fractal dimension-sparsity curve based on the line chart above by using least 
square method.

According to Figure 3, we assume that fractal dimension and sparsity has one degree linear relationship: $K=\alpha \cdot f+\beta$. $K$ represents estimated sparsity value and $f$ represents fractal dimension which will be estimated. Different curves simulated by Matlab poly fit and least square method are given below. The estimated formula of fractal dimensions and image sparsity is given below as well.

$$
\mathrm{K}=211.6471 \cdot \mathrm{f}-514 \text { : }
$$

Curve plot is shown in Figure 3(b), Table 1 shows calculation results of 6 samples:

Table 1. Observation matrix size(SD) and fractal dimension(FD)

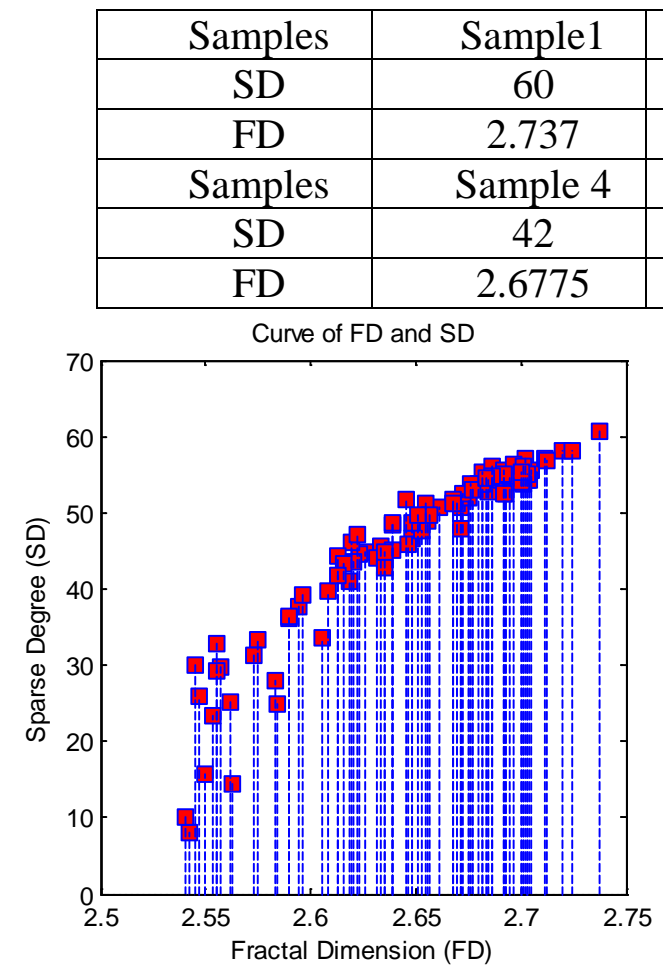

(a)

\begin{tabular}{|c|c|}
\hline Sample 2 & Sample 3 \\
\hline 56 & 56 \\
\hline 2.697 & 2.7012 \\
\hline Sample 5 & Sample 6 \\
\hline 36 & 36 \\
\hline 2.5532 & 2.5839 \\
\hline
\end{tabular}

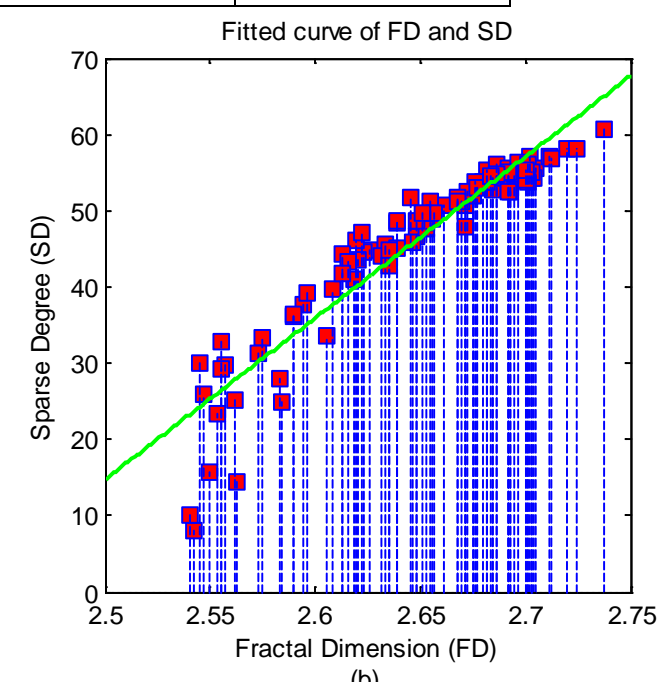

(b)

Fig.3. Fitted curve of fractal dimension and sparsity degree

\section{Verification of sparsity estimation algorithm}

- Verification of algorithm 1:

More samples are chosen to verify equation (3) as following:

1. Calculate the fractal dimensions of samples

2. Use equation (3) to estimate sparsity $\mathrm{K}$. When estimation value is not a integer, take the smallest integer that is bigger than the value.

3. Use DCT as base function and random matrix as measurement matrix to compress sample images.

4. Use OMP algorithm to reconstruct and calculate the signal-noise ratios of reconstructed images.

By using previous steps, the experiment results in table 2 shows that sparsity estimation method of SAR images has very high accuracy.

Table 2. Validation results

\begin{tabular}{|c|c|c|c|c|}
\hline Samples & Sample 1 & Sample 2 & Sample 3 & Sample 4 \\
\hline FD & 2.7495 & 2.6048 & 2.6828 & 2.5975 \\
\hline SD & 68 & 40 & 56 & 36 \\
\hline $\begin{array}{c}\text { Reconstruction } \\
\text { SNR }\end{array}$ & 21.5487 & 21.5502 & 20.3893 & 20.0388 \\
\hline
\end{tabular}

Also sparsity-signal noise ratio simulations on many samples have shown that the signal-noise 
ratio and sparsity has increment relationship, as curves shown in Figure. 5. Red bold curve is the result of simulation and the increment relationship is shown in equation (4). Then according to equation (4), the result can be modified when the signal-noise ratio of reconstructed image changes. In conclusion, equation (3) gives out the sparsity estimation algorithm when signal-noise ratio is $20 \mathrm{db}$ and equation (4) gives out the modification of sparsity estimation when signal-noise ratio is not 2-db.

$$
\Delta \mathrm{k}=0.6218 \times \Delta \mathrm{psnr}
$$

\section{- Verification of algorithm 2:}

In this paper, the result estimated by the method above also needs to be verified with traditional sparsity estimation method. Sparsity is usually defined as the estimation value of non-zero vectors in a matrix. More precisely, verification will base on comparing the ratio of non-zero coefficients in a matrix and the ratio between the length of measurement vector and original sample size.

The estimation algorithm proposed in this paper will be compared with DCT traditional estimation algorithm. DCT estimation has following steps: first DCT coefficients matrix will be resulted from applying DCT transformation on images, then calculate the non-zero coefficients in the matrix treating small values as zeros, at last calculate the ratio between the value and total coefficient of the image.DCT coefficients are calculated on all 88 sample image, meanwhile the calculated sparsity value $\mathrm{K}$ is transformed into the length $\mathrm{M}$ of the measurement matrix and then the ratio between $\mathrm{M}$ and original length will be calculated. As shown in Figure 4:

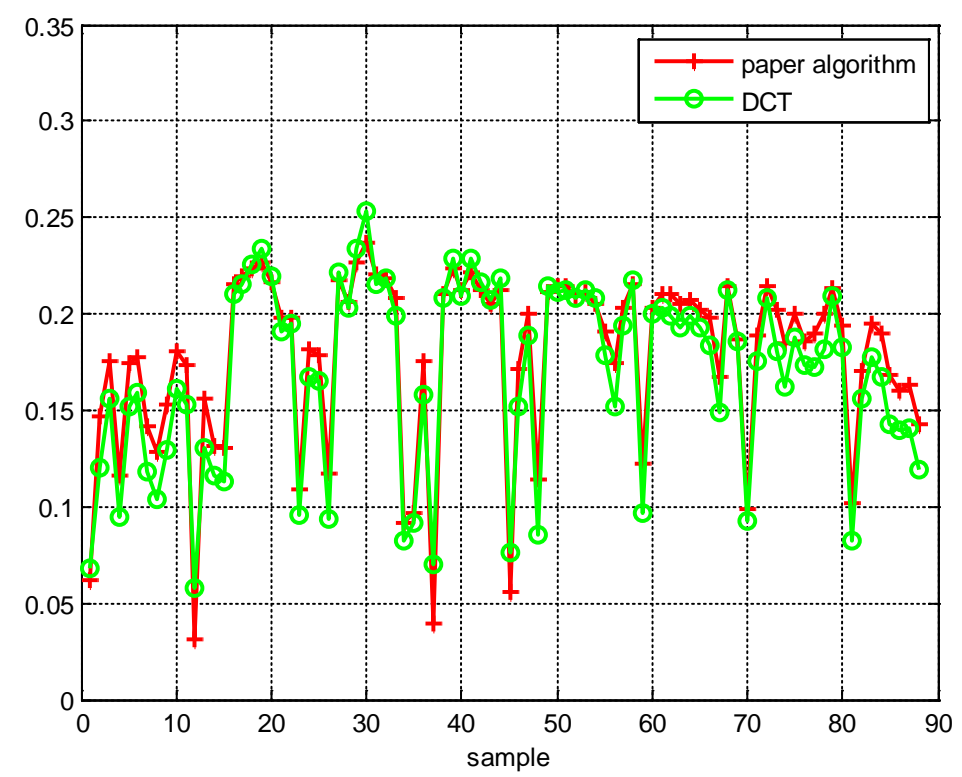

Fig.4. Compare of our algorithm and DCT transform method

According to Figure.4, the ratio of non-zero coefficients based on DCT transform is very similar to the estimation of the sparsity given in the paper. Here the DCT threshold value of non-zero coefficients is made based on the error of reconstructed images which is less than 5\%. Different threshold values will have different results. However, the patterns of curves are very similar.

\section{Conclusion}

Due to the importance of parameter $\mathrm{M}$ in measurement matrix while applying estimation of sparsity of image in Compressive sensing, this paper proposes estimation on calculating sparsity of SAR image based on fractal dimensions. Because fractal dimension represents the pattern of images, we assume that there exists bijections between fractal dimensions, sparsity of images and entropy of images. The linear relationship between fractal dimensions and signal-noise ratio of reconstructed image is found by examining 88 sample images by computer simulation, thus the assumption above is valid and the calculation result of fractal dimension can be used to estimate the sparsity $\mathrm{K}$ of images. This paper also uses simulation results to simulate the linear relationship between fractal 
dimensions and sparsity $\mathrm{K}$ under given signal-noise ratio (the ratio is $20 \mathrm{db}$ in this paper). Such function can decide a suitable parameter $\mathrm{K}$ of measurement matrix from calculating the fractal dimensions of images. The function shows that taking $\mathrm{M}=4 \mathrm{~K}$ is a suitable parameter for the matrix. In the end, the algorithm in this paper is also examined by computer simulation. The result shows that the algorithm has high accuracy on estimation of value of $\mathrm{M}$ and the complexity of such algorithm is lower than complexity of other algorithms.

\section{Acknowledgement}

In this paper, the research was sponsored by Science \& Technology Program of Shanghai Maritime University (Project No. 20120066).

\section{References}

[1] Candes EJ, Romberg J, Tao T. Robust uncertainty principles: Exact signal reconstruction from highly incomplete frequency information[J]. IEEE Trans Inf Theory. 2006, Vol.52(2):489-509.

[2] Duarte M F and Baraniuk R G. Kronecker compressive sensing[J]. IEEE Transactions on Image Processing, 2012, 21(2): 494-504.

[3] TsaigY, Donoho D. Extensions ofcompressed sensing[J]. Signal Process.2006, Vol.86(3):549.

[4] Ma Yuan, Lu Yang-Yang, Qian Lu-Lu, Pei Lin-Lin, Image sparsity evaluation based on principle component analysis, Acta Physica Sinica, 2013 Vol.62(20): 204202.

[5] KohonenT. Improves Version of the Learning Vector Quantization[C]. Proc of the IntConf on Neural Networks. 1990.545-550.

[6] Gao Min. SAR object recognition compressed sensing based. Master’s thesis, 2010.

[7] He Chu, Liu Ming, Feng Qian, Deng Xinping. PollnSAR image classification based on compressed sensing and multi-scale pyramid[J]. Acta Automatica Sinica, July 2011, vol.37(7): 820-827. 